\title{
BMJ Open Knowing what the patient wants: a hospital ethnography studying physician culture in shared decision making in the Netherlands
}

\author{
Laura Spinnewijn (D) , ${ }^{1}$ Johanna Aarts, ${ }^{1}$ Sabine Verschuur, ${ }^{2}$ Didi Braat, ${ }^{1}$ \\ Trudie Gerrits, ${ }^{2}$ Fedde Scheele ${ }^{3,4}$
}

To cite: Spinnewijn L, Aarts J, Verschuur S, et al. Knowing what the patient wants: a hospital ethnography studying physician culture in shared decision making in the Netherlands. BMJ Open 2020;10:e032921. doi:10.1136/ bmjopen-2019-032921

- Prepublication history and additional material for this paper are available online. To view these files, please visit the journal online (http://dx.doi. org/10.1136/bmjopen-2019032921).

Received 15 July 2019 Revised 15 January 2020 Accepted 07 February 2020

Check for updates

(C) Author(s) (or their employer(s)) 2020. Re-use permitted under CC BY-NC. No commercial re-use. See rights and permissions. Published by BMJ.

${ }^{1}$ Obstetrics and Gynaecology, Radboudumc, Nijmegen, The Netherlands

${ }^{2}$ Faculty of Social and

Behavioural Sciences, University of Amsterdam, Amsterdam, The Netherlands

${ }^{3}$ Department of Research and Education, OLVG Hospital, Amsterdam, The Netherlands ${ }^{4}$ Faculty of Earth and Life Sciences, Athena Institute, VU University, Amsterdam, NorthHolland, The Netherlands

Correspondence to Laura Spinnewijn; lauraspinnewijn@gmail.com

\section{ABSTRACT}

Objectives To study physician culture in relation to shared decision making (SDM) practice.

Design Execution of a hospital ethnography, combined with interviews and a study of clinical guidelines. Tenweek observations by an insider (physician) and an outsider (student medical anthropology) observer. The use of French sociologist Bourdieu's 'Theory of Practice' and its description of habitus, field and capital, as a lens for analysing physician culture.

Setting The gynaecological oncology department of a university hospital in the Netherlands. Observations were executed at meetings, as well as individual patient contacts.

Participants Six gynaecological oncologists, three registrars and two specialised nurses. Nine of these professionals were also interviewed.

Main outcome measures Common elements in physician habitus that influence the way SDM is being implemented. Results Three main elements of physician habitus were identified. First of all, the 'emphasis on medical evidence' in group meetings as well as in patient encounters. Second 'acting as a team', which confronts the patient with the recommendations of a whole team of professionals. And lastly 'knowing what the patient wants', which describes how doctors act on what they think is best for patients instead of checking what patients actually want. Results were viewed in the light of how physicians deal with uncertainty by turning to medical evidence, as well as how the educational system stresses evidence-based medicine. Observations also highlighted the positive attitude doctors actually have towards SDM.

Conclusions Certain features of physician culture hinder the correct implementation of SDM. Medical training and guidelines should put more emphasis on how to elicit patient perspective. Patient preferences should be addressed better in the patient workup, for example by giving them explicit attention first. This eventually could create a physician culture that is more helpful for SDM.

\section{INTRODUCTION}

Over the past decades, societal developments have propelled the interest in shared decision making (SDM) in medicine. ${ }^{1}$ Treatment choices are no longer solely made by doctors,
Strengths and limitations of this study

- Hospital ethnography produces valid data and is particularly useful for interpreting data within social contexts.

- The French sociologist Bourdieu's theorem of habitus, field and capital provides a helpful lens for analysing physician culture.

- The insider/outsider construction used in this study provided quick and easy access to the field and reduced bias due to subjective reporting.

- Results in this study are not directly generalisable to other medical fields or environments, as no two contexts are the same, however, the insights gained may inform the understanding of comparable dynamics in similar settings.

but by involving patients in this process. ${ }^{2}{ }^{3}$ Healthcare has thus moved from a primarily doctor-centred system towards a more patientcentred system. SDM is an approach that involves at least two participants: the patient and the healthcare provider. Both parties actively participate in the decision-making process. They share information before reaching consensus on treatment choices to be made. ${ }^{1}$ This short definition, however, only partially addresses the complexity of the SDM process. According to Charles et al, SDM requires a doctor to establish an atmosphere for patients to share their views, followed by physicians actively eliciting patient preferences. Physicians should subsequently provide information on treatment options, risks and benefits, appropriate for this specific patient (p687). ${ }^{1}$ To complicate matters, it is often not clear how many or what type of information a patient wants. Therefore, SDM requires doctors to actively explicate patient needs, before options are discussed and decisions are made. ${ }^{1}$ 
Contemporary research has explored several important issues relating to the implementation of SDM. Examples are the definition of required competencies for doctors in implementing SDM principles, ${ }^{4}$ the identification of barriers for implementation ${ }^{5}$ and the development of decision aids. ${ }^{6}$ Health outcomes have been investigated as well. Patients, for example, show better adherence to treatment, and both patients and healthcare professionals show more satisfaction when using $\mathrm{SDM}^{7}$ This could potentially lead to more positive effects on diseaserelated outcomes as well, ${ }^{7}$ although empirical research to prove these effects is lacking. ${ }^{8}$

Despite all the efforts to implement SDM in medical practice, it has never reached the desired level of embedment in usual patient care. ${ }^{8}$ Practitioners, for example, only partially and inconsistently use decision aids correctly. ${ }^{9}$ Furthermore, in decisions to use highimpact treatment modalities like chemotherapy, deliberation and joint preference construction are not standard practice. ${ }^{10}$ Physicians even have shown resistance to the changes required to execute SDM correctly. ${ }^{811}$

The reasons why doctors are reluctant to implementing changes, and SDM in particular, are not fully understood, yet research indicates physician culture, expressed in a set of shared beliefs, knowledges and practices, might be of great importance. ${ }^{11}$ However, studies into physician culture are rare. One reason for neglecting culture in SDM research may be that the sociocultural environment is difficult to grasp. Its presence is rather implicit, with complex interactions and vague descriptions. The use of scientific methodology from social sciences could help us to better understand the concept of culture, as it provides notions and insights on how to explain its presence.

This study uses the work of French sociologist Pierre Bourdieu and his concepts of habitus, field and capital as a lens for explicating physician culture relating to SDM principles. ${ }^{12}$ An ethnographic study was performed, in which elements were identified that might predict or even stipulate physician behaviour in SDM practice. It focused especially on those elements that could explain the incorrect execution of SDM. The study was executed in a gynaecological oncology department. Oncology care is believed to be challenging in implementing SDM, since this field is particularly regulated and guideline driven. ${ }^{13}$ New insights from this study could potentially be useful to redesign SDM implementation processes and further improve decision making in healthcare.

\section{METHODOLOGY}

\section{Setting}

In contrast to other countries, the Dutch healthcare system is not divided into private and public healthcare. All Dutch citizens have mandatory health insurance, and essential healthcare is paid for by the insurance. ${ }^{14}$ This makes oncological care easily accessible for everyone.

The government has played a crucial role in how healthcare is currently shaped. Over the past decades, the Dutch government has designed laws and regulations to make healthcare more patient oriented and demand driven (p.284). ${ }^{3}$ Consumer experiences are highly valued and several government initiatives are employed to encourage patients to engage in their medical treatment decision process. ${ }^{15}$

The study at hand was conducted within the gynaecological oncology department of an academic centre in the Netherlands where gynaecologists dedicate their work solely to the treatment of gynaecological cancer. A large part of patients are referred by neighbouring hospitals to receive specialised care.

\section{Research methods: hospital ethnography}

For this study, hospital ethnography was used to collect data. ${ }^{16}$ Ethnography is a qualitative research method specifically aimed at studying culture and has its origin in anthropology. It is characterised by often longer periods of so-called participant observation in a natural setting, combined with other ways of qualitative data collection. ${ }^{17}$ In participant observation one aims to gain close contact with a group in their cultural environment. Participant observation is crucial in ethnography, because it "provides access to what people do and what they say about it, instead of only listening to what people say that they themselves or others do' (p32). ${ }^{18}$

Observations were performed by two researchers (LS and SV). The first had experience in the field of gynaecological oncology as a registrar in obstetrics and gynaecology. The second was a student in medical anthropology. In the initial phase of observations and data processing they received strict supervision from the medical anthropologist (TG), who has extended experience in hospital ethnography. ${ }^{18}$ The level of supervision decreased with each sequential phase. Observations took place over a period of 10 weeks, from March to May 2018. Both researchers were each approximately 2-3 full working days per week present performing participant observations. An exploratory approach was used, where observations were combined with interviews. All interviews were held by the second researcher in the last 4 weeks of the study period and included both formal and informal interviews.

The main focus of observations was on a team of six gynaecological oncologists, three registrars and two specialised nurses. The researchers observed various group specialist meetings, as well as individual doctorpatient encounters.

Field notes were taken during observations and processed afterwards. ${ }^{19}$ Field notes were discussed with the project team at least every 3 weeks.

Informed consent for observations and the use of data was received from all participants prior to conducting the observations and interviews. Participants were given identity codes to preserve anonymity.

\section{Data collection and analysis}

Data collection, processing and analysis were done simultaneously throughout the whole observation period. 
Emerging themes from observations and interviews were used to structure future observations and activities. ${ }^{17}$ Data collection was divided in three phases, each phase containing at least one team meeting (LS, SV, TG, JA, FS and DB; in changing group compositions).

In phase 1, the researchers introduced themselves to the field and performed exploratory observations. ${ }^{17}$ Most observations during this phase were done simultaneously (LS and SV). Field notes were thematically coded by the researchers separately (LS and SV). Next, notes and themes were compared and subsequently refined in a code list through an iterative process. This list was then used to design more structured observation forms, to be used in the next phase.

In phase 2, data were collected with the help of these forms (see Observations forms phase 2 in online supplementary appendix 1). Both researchers now started performing separate observations. Furthermore, a semistructured interview guide was developed, reflecting some previously emerged themes from phase 1 . The interview guide contained questions on the use of SDM, as well as two hypothetical clinical cases, and is added in online supplementary appendix 2 . For the cases, the interviewee was asked to elaborate on two fictional patient workups, including their decision-making process.

After discussing first results at the start of phase 3, the observation forms were redesigned, including a checklist with key elements in SDM, ${ }^{20}$ as well as a checklist on interprofessional attitudes to aid the interpretation of observations (see checklists added in online supplementary appendix 3). ${ }^{21}$ Next, final observations and interviews were performed. A total of nine formal interviews were conducted, with five gynaecologists, two registrars and two specialised nurses.

Finally, national guidelines for gynaecological oncology care were examined, focusing on the presence of SDM principles. $^{22}$

At the end of data collection, all observational notes and interview transcripts were thematically analysed and discussed in a team meeting.

\section{Bourdieu's theory of practice}

This study starts with the assumption that medical culture influences the practice of SDM, and that physicians are socialised in this culture through formal and informal ways. It uses Bourdieu's 'Theory of Practice', with its concepts of habitus, field and capital, to explicate physician culture and to understand how people bidirectionally influence and are influenced by their environment. ${ }^{12}$

Habitus has been used before to study medical culture. Hightower, for example, studied physician culture at an emergency department. ${ }^{23}$ She defines habitus as 'all actions, behaviours, and responses performed by the emergency physician as a part of their day-to-day routines' (p.2) ${ }^{23}$ Habitus, thus, refers to individual views, beliefs and actions, yet it places those elements in a social context as well. ${ }^{24}$ People's experiences within a social environment actually become embodied in their habitus. ${ }^{24}$ In a medical context, this means that physicians' habitus is influenced, among other things, by medical training, interactions with colleagues and patients, and the rituals and routine in a medical department (p.31). ${ }^{23}$ No two habitus will be exactly the same, but within the same social group they will share similarities, as two individuals are influenced by the same external structures. In fact, harmonisation of habitus between individuals belonging to one cultural group confirms the embodied group structure. ${ }^{12}$ Habitus in turn also shapes the system by reproducing all the group rules or rituals. ${ }^{23}$ A change in habitus, therefore, also influences the environment. Habitus can change, as it evolves in interaction with changing individual treats and external influences. People will unconsciously act according to their habitus, developing new and different strategies as they travel through various social fields. ${ }^{25}$

The second concept, 'field', is inevitably interconnected with habitus and can be described as the social environment of the individual, with its own rules of practice. Where habitus is the 'feel for the game', field provides the 'rules of the game'. ${ }^{24}$ Field could refer to a social field, like a healthcare field such as gynaecology, or to another social structure where a certain distribution of power leads to contesting forces. ${ }^{25}$

The third element, 'capital', stands for all the resources an individual has at his disposal, including social, cultural, intellectual and economic capital. ${ }^{24}$ Skills and knowledge, for example, can be considered capital. Capital resources can be used both consciously and unconsciously.

Bourdieu considers the three elements as interrelated elements, which together may predict practice to a large extent. This interconnection implies that altering any of the elements could consequently create a different practice. ${ }^{26}$ It explains both differences in practice between individuals within the same social context (as their habitus differs at some points), and the different actions from one individual in different settings (as the field changes and different capital resources might be exploited).

\section{Patient and public involvement statement}

No patients or public were involved in the design of this study.

\section{RESULTS}

As with all hospital ethnographies it is not possible to fully describe all observational results. Physician practice is, therefore, illustrated by picturing a typical patient workup at the gynaecological oncology department (see box 1). The case is fictional, yet it combines elements from real observations to illustrate practice and to provide a background for the rest of the results description. Figure 1 subsequently shows key features of gynaecological oncologists' habitus, capital and field, identified in this study. These particular features resulted from the thematic analysis, and are selected as they seem related to the way SDM was executed. The three elements from doctors' habitus are further described in our results section, as they refer 
Box 1 A typical patient workup at the gynaecological oncology department

Tuesday morning 9:30; the weekly meeting to discuss all referrals and planned surgeries starts. Five gynaecological oncologists, two registrars and one nurse are present. First, all upcoming surgeries are discussed and afterwards all new patient referrals, planned for the afternoon, are presented by one of the registrars.

One of the new patients, mrs X, is a 50-year-old woman, diagnosed with vulvar cancer. Information available from the referring hospital is presented, mainly consisting of biopsy results and characteristics of the vulvar lesion. The presenting registrar is questioned about the reigning guidelines on diagnostic workup and treatment of vulvar malignancies, and a preliminary plan for workup and treatment is briefly discussed. Both registrars and gynaecologists seem well informed about the guidelines, and they quickly continue discussing the next patients. There is not much time, as this meeting will be immediately followed by the weekly multidisciplinary tumour board meeting and must therefore finish on time.

The tumour board meeting starts at 11:00 sharp. All previously mentioned professionals are present, supplemented by an oncologist, radiologist, pathologist and radiotherapist. There is also an 'audience' present, consisting of five researchers working in the field of medical oncology. At the tumour board meeting, results from diagnostic and therapeutic procedures are discussed. The multidisciplinary team then formulates a recommendation for treatment for a particular patient. Most recommendations are formulated in accordance with current guidelines or medical evidence from contemporary medical trials. The tumour board meeting is characterised by a high pace, since results from 30 to 40 patients are addressed each week.

A few other hospitals are presenting their cases in this meeting as well, through video conferencing. Mrs $X$ is now presented by the referring hospital, focusing on pathology results and the presumed following diagnostic and therapeutic procedures.

It is now 13.30 and mrs $X$ is now consulting her physician. She heard only a week ago that she suffered from a malignancy and is very emotional. She says that she is devastated and that she doesn't understand why this is happening to her. 'I just had my breasts examined and they were fine!'. Her husband accompanies her, and while she is crying, he mainly is quiet. The woman states that she keeps thinking the worst will happen to her and that she 'does not want to know everything'. The consulting physician, who is the same registrar presenting in the morning meeting, tries to reassure her by explaining what will happen today and the following weeks. Next, the patient's history is discussed, followed by a physical examination.

During the examination, one of the gynaecologists joins the consultation. Afterwards, the registrar explains to the couple, together with the gynaecologist, what the treatment plan will be. 'Our advice would be to perform surgery. There is a tumour present, but it will not be difficult to remove. During the surgery we will also remove lymph nodes from the groin.' The registrar gives a technical explanation of the procedure and possible complications. Mrs X asks many questions, for example, 'What if I don't wake up from the anaesthetic?' and 'What if they can't find the right lymph node?' The registrar tries to reassure the patient, 'The chances you be cured are really good'. 'I trust you', the patient finally states.

At the end of this consultation, which took about $45 \mathrm{~min}$, mrs $X$ is seen by a nurse, who provides more information about the procedures. She gets an appointment for an ultrasound, a meeting with the anaesthesiologist, and ultimately her surgery is scheduled.

\section{Box 1 Continued}

At the end of the afternoon a debriefing takes place, with all registrars and gynaecological oncologists present. Again, all new patients are discussed, including mrs $X$. The registrar briefly describes her workup, now also mentioning that the patient was very emotional. One of the gynaecologists asks the registrar what she would do if the tumour was slightly different in size. When the registrar does not give the 'right' answer, she is told to look it up again in the guideline.

most to doctors' practice. Capital and field will not be elaborated on as separate themes in this results section.

\section{Emphasis on medical evidence}

During the observations, it became apparent that medical evidence, mostly obtained from clinical trials, played a key role in the doctors' daily practice. This fits well with elements from capital and field. The Dutch medical training system is based on the principles of evidencebased medicine (EBM), and gynaecological oncology is a strictly regulated field of care.

Especially in tumour board meetings many references were made to results from medical studies and to evidence-based guidelines, mainly to determine the 'right' diagnostic procedure or treatment protocol for an individual patient. The patients' wishes and perspectives were sometimes explicitly mentioned but were not given structural attention.

When doctors and nurses were asked to comment on the lack of patient perspective in board meetings, reasons mentioned were lack of time, that the meetings were meant for representing 'medical data', and that according to them the patient perspective was sufficiently represented in these meeting through the presence of a nurse as the patient's case manager. In practice, however,

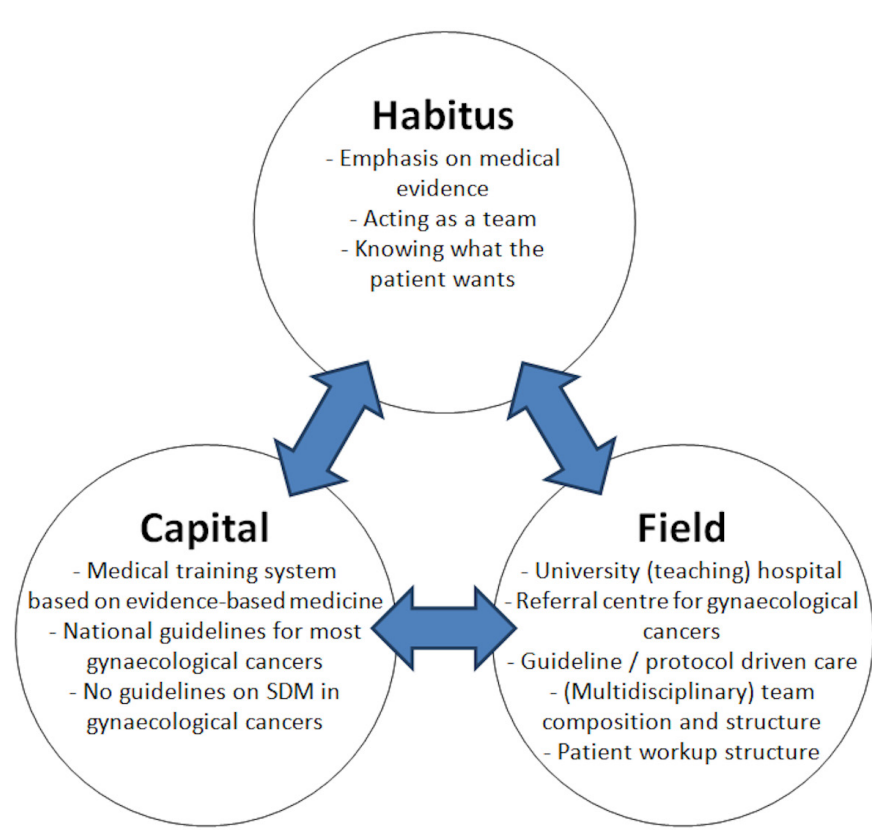

Figure 1 Physician culture: key features of habitus, capital and field. SDM = shared decision making. 
these nurses rarely contribute to the discussion. As one of the nurses stated:

'During tumour board meetings we are always in a hurry, so you can't elaborate on every patient. So, yes, the conclusion often is a medical conclusion. Context is eventually discussed in the conversation with the patient.' (Source: interview with a specialized nurse).

In doctor-patient encounters, the same tendency to emphasise medical evidence was observed. Although patients were asked about their perspectives most of the time, first encounters often still emphasised 'what is best practice' in terms of treatment decisions based on guidelines and other medical evidence. In the case described in Box 1, for example, no alternatives were discussed besides from the workup advised within the guideline for treating vulvar cancer.

However, patient encounters following initial treatment were much less focused on medical evidence and executing protocol led care, and provided more room for patient perspectives.

A young woman comes in for a follow-up visit, 1.5 years after the initial treatment for a gynaecological cancer. She asks the doctor: 'Wouldn't it be valuable for me to have a scan so I can feel reassured?' After initially explaining that reigning guidelines state that scans do not contribute to a 'good workup', doctor and patient still agree on performing a scan, since they both think this would reassure the patient. (Source: observation of a patient-doctor encounter).

In this case, no guideline prescribed the extra check, yet the doctor listened to the patient's wishes and together they agree on performing a scan. This conversation is exemplary for how the gynaecological oncologists tended to respect patient perspectives within their clinical encounters, when brought up. Observations showed that especially when palliative care was indicated, patient wishes were elicited more often than when cure still seemed possible.

The emphasis on medical evidence was also visible beyond formal meetings and patient visits. The hospital where this study was executed is a teaching hospital where both medical trainees and registrars in gynaecology are trained. This training clearly emphasised medical knowledge. Registrars were for example actively encouraged to familiarise themselves with reigning guidelines for oncological care, as seen in Box 1, but not structurally coached on how to deal with patient wishes. Furthermore, the specific guidelines for gynaecological oncology care did not incorporate patient perspectives in the diagnostic workup and showed only minor variation in treatment advice based on patient characteristics such as age and comorbidity. During the study period, no guidelines existed on how to deal with patient preferences or SDM. Furthermore, no patient decision aids to facilitate SDM were in use, although the department was in the procedure of developing a decision aid for patients with advanced ovarian cancer.

\section{Acting as a team}

Doctors often tried to reach consensus on their joint medical advice for a patient before meeting that same patient, as observed in the morning meeting and in the tumour board meeting. Especially in cases where medical guidelines were not readily available. This group advice had a prominent place in the subsequent patient consultation. As one of the gynaecologists phrased:

Interviewer: 'How does reaching consensus influence the way you give medical advice to the patient? Or doesn't it influence your actions?' Doctor: 'Yes, it does have influence, because if we've reached consensus in the meeting, I will tell the patient:

'This is the advice of our tumour board'. (Source: interview with a gynaecological oncologist).

In general physicians often presented themselves in patient encounters as representatives of a team of doctors. They even tended to speak in terms of 'we' rather than 'I'. In the case described in Box 1, the registrar, for example, referred to 'our advice', instead of to 'my advice'.

The following quote is another example of how the team's voice was presented in a patient encounter. It also illustrates how the group recommendation is presented to the patient first, before eventually tuning in on the patient's perspective.

A patient who is currently being treated with chemotherapy for ovarian cancer checks in for a follow-up visit. Doctor: "When we consider surgery, like in your case, we look at three important issues. First, what we see on the scan, which has been positive since the start of your chemotherapy. Next we look at your blood values, which have improved. And lastly, how you feel. We think it's better for you to have the surgery. But we also want to know how you feel'. (Source: observation of a patient encounter)

\section{Knowing what the patient wants}

Doctors often seemed to think they knew what was best for their patients. On the one hand this might be true, since they seemed very involved with their patients. On the other hand doctors often filled in patient's wishes beforehand, based on clinical patient characteristics and without explicitly asking what the patient wants, especially in first patient encounters. This may have to do with logistics around newly referred patients, as the case in Box 1 also illustrates. The hospital under study is a referral centre for the treatment of gynaecological malignancies, so part of the diagnostics are usually performed elsewhere. As one nurse describes:

'When people are referred by another hospital, we already start to plan their treatment. And when we start acting, we've only seen them once. So how well do you know someone?'. (Source: interview with a specialised nurse)

The first consultation in the hospital under study often concluded with determining next steps in the diagnostic workup, or even definitive treatment planning, and often lacked a full exploration of treatment options. For example, the option 'no treatment' was rarely given. One doctor commented that patients would not have been referred if they did not want any treatment, but 
admitted to not always check whether this actually was the case.

The following example illustrates how treatment decisions may lead to an unwanted result:

Nurse: 'I once spoke with a woman who had (major surgery). (...) She said: 'If I had known this beforehand, I would never had initiated the surgery.' It's difficult for people to imagine what it does to you when you're in the midst of it. Because you don't know what it does to a patient's life.' (Source: interview with a specialized nurse)

This demonstrates how hard it is to predict what impact certain treatment modalities have. However, eliciting patient wishes and truly knowing what is important in a patient's life might help the doctor in picturing how the chosen treatment influences a patient afterwards, and so improve SDM before initiating this treatment.

One example of how the department did explicitly incorporate patient perspective, was the so-called 'complication meeting'. This was a meeting where a specific complication that occurred to a patient was analysed. The affected patient was present at this meeting and had the opportunity to elaborate on her experiences. Next, healthcare professionals analysed the incident and arranged improvements in patient care to prevent future harm.

This dedication to do well for patients was also visible at other times. Apart from first encounter visits, in general the doctors knew and cared about patients' well-being and social situation. After initial treatment, they showed great attention for patients' wishes, needs and struggles in daily life. We observed many doctors openly reflect on their own thoughts and emotions, and on the dreadful consequences of patient's severe illnesses. They seemed dedicated to guide their patients the best they could, both medically and in other aspects of life, and they put in extra effort to do so.

'Sometimes I call the general practitioner (of a patient) to tell him about the medical situation and to ask: 'You know this lady better than I do. This is the situation, how can I best deal with this?' (Source: interview with a gynaecological oncologist)

\section{DISCUSSION}

\section{Statement of principal findings}

This study describes three elements in doctors' habitus that might affect the way SDM is handled. These elements are: the strong emphasis on medical evidence in decision making; the fact that doctors present themselves in their conversations with patients as a whole team instead of as an individual doctor; and that doctors seem to act on what they think is best for patients, instead of primarily informing themselves on what patients truly want and need. In the following section the authors will reflect on those elements.

Why do doctors emphasise medical evidence, instead of focusing on individual patients' needs first? One reason might be the rise of EBM, which has profoundly changed medicine since its origin some 25 or more years ago. The way EBM is used in this study setting, however, is not in line with the initial idea behind EBM. Sackett $e t$ al already explained in 1996 that EBM not only consists of the best 'external medical evidence' obtained from systematic research, but also of the 'identification and passionate use of individual patients' predicaments, rights, and preferences' (p.71). ${ }^{27}$ So from this perspective EBM is not merely about applying medical evidence, but it requires doctors to adjust this evidence to individual patient situations. In the current study setting, this is what frequently seemed to be lacking, especially in group specialist meetings and in first patient encounters, as treatment advice was made before or without actively eliciting patient wishes.

Another explanation for the emphasis on medical evidence may lie in the way doctors deal with uncertainty. Oncology care has a high level of uncertainty, and although doctors are aware of this uncertainty it is in their nature to suppress it. ${ }^{28}$ Medical training starts building this rationalist medical approach, as junior doctors are being trained to execute several examinations and tests, to subsequently determine the right and neatly labelled diagnosis. So young doctors learn to seek apparent security in data and algorithms, instead of learning other ways to handle uncertainty. ${ }^{28}$ The same educational capital is visible in the current study setting, with registrars being strongly encouraged to profoundly familiarise themselves with medical evidence, while they are hardly encouraged to learn how to deal with individual differences in patient needs and wishes or to gain expertise on communication skills to elicit patient wishes.

Another study on culture among physicians showed that medical trainees even learn to avoid uncertainty. It showed that socialisation in surgical culture moves students towards traits that mask uncertainty and consolidate status. ${ }^{29}$ Even though this was not apparent in the department under investigation in this study, this is exemplar for the absence of apt ways to deal with uncertainty.

Reaching group consensus can be considered as another approach to deal with uncertainty. Being confronted with the advice of a 'team of experts' first, however, limits patients in being a true partner in the decision-making process, as patients are in a vulnerable position in a to them unfamiliar domain. ${ }^{30}$ The 'strong voice' may help in, for example, reassuring patients, but because the decision making in the team has already been done, it might also hamper true shared decision-making when presented to patients first.

In this study, no patients were present when the team advice was formulated. This phenomenon has been studied by others as well. ${ }^{31}$ T2 The authors from the current study do not want to plea for patient presence at tumour board meetings. However, knowledge of patients' individual needs during group specialist meetings might already in this stage prepare for SDM.

Doctors in the current study in general were positive about addressing patient wishes, but again the field did not help them. As shown in box 1 high work pace was 
observed, especially at group specialist meetings, with individual patients being often discussed in under $2 \mathrm{~min}$. Furthermore doctors made decisions about treatment programs at a time when they did not yet know their patients well, in both first patient encounters, as well as in group meetings. Literature shows that in these types of situations doctors seem to base decisions more on superficial interactive knowledge and stereotypes, and they tend to apply their own value judgements more. ${ }^{33}$ When gynaecological oncologists knew their patients better, care was tailored more towards patient preferences. ${ }^{33}$ Another observational study of tumour board meetings showed that patient characteristics were mentioned more frequently during those meetings where more time was spent discussing an individual patient. ${ }^{32}$ The current study setup is not designed to show such causalities. In the light of these previous study results, however, it is plausible that the way patient workup was organised might be limiting the proper use of SDM principles.

Another study suggests a need to explicitly voice patient preferences. It showed that in a minority of oncology consultations for rectal cancer this actually happened, yet whenever it did, this increased patients' perceived involvement in the decision-making process significantly. ${ }^{34}$

\section{Strengths and weaknesses of this study}

A strength has been that this was an observational study, so this study is able to report on what doctors actually do rather than what doctors say they do.

Hospital ethnography is a scientific approach tailored to study culture and thus it is the appropriate method for answering the research question. As in most qualitative research there is, however, a certain level of subjectivity, due to the personal impact on what is observed, noted down and interpreted. In this study, this was compensated by using an insider-outsider construction. This proved to be a great advantage. The insider perspective facilitated quick and easy access to the work field. It also facilitated understanding of medical jargon and processes and accelerated the establishment of a relationship between field and researchers. While the insider might have been cultivated too much in training, the outsider compensated this by being new to the field of medicine. Furthermore, an experienced hospital ethnographer was used to supervise the process and to help interpret findings and minimise bias.

Data obtained in this study were linked to existing literature on culture and SDM throughout the data collection. Furthermore, a well-established social sciences theory was used, which made it easier to analyse features of physician culture. Bourdieu has been used before in describing cultural elements in healthcare. ${ }^{35}$ What's new in this study, however, is that his theory is applied to analyse culture among doctors specifically.

A big disadvantage of observational studies in general is the lack of generalisability of study results, as phenomena observed seem legit for the field under observation, but not necessarily are so for all other fields. However, there might still be a generalisability of concepts,${ }^{36}$ as field and capital share similarities between different hospitals. Therefore, insights gained in elements from doctors' habitus-and how this may affect SDM-in this study could still enhance understanding of similar dynamics in other situations as well.

\section{Strengths and weaknesses of this study in relation to other studies}

To the best of our knowledge, this is the first observational study that evaluates physician culture in relation to SDM. Other types of studies looking at physician culture in relation to physician practice are scarce and therefore it is hard to compare results or even find similarities across studies. The previously mentioned study on surgical culture, for example, paints a more negative picture than our study ${ }^{29}$ with traits like harshness and invulnerability, which we did not recognise in our study group.

One interview study among obstetricians confirmed that the way healthcare is organised could potentially hamper SDM. It demonstrated that fragmented and emergency care, with little time for doctors getting to know their patients, sometimes leads to misjudging patient preferences. ${ }^{33}$ This could explain why care is tailored more to individual differences later on in the patient workup, when doctors got to know their patients better, as was observed in the current study.

Another report on the same interview study showed that obstetricians felt confident to base their decisions on their own clinical expertise and patient choice, instead of adhering to guidelines. ${ }^{37}$ However, it also reported reasons for doctors to adhere to guidelines instead, even in situations where they did not want to. Reasons mentioned were pressure from colleagues or a fear of lawsuits. ${ }^{37}$ The fear of lawsuits was not observed in the current study, yet this could vary from country to country, as the respective judicial systems (e.g. the judicial capital) differ.

\section{Meaning of the study}

The novelty in this study is that a different approach was used to assess SDM execution in practice. Understanding how physician culture, defined by habitus, field and practice, has its effect on doctors' behaviours, provides new clues on how to change practice towards better execution of SDM principles. One suggestion would be to give explicit attention to patients' preferences in the early stages of patient workup by spending more time to get to know the patient before treatment advice is discussed. Tumour board meetings and other team meetings should be reviewed and redesigned to support both SDM and EBM principles, for example, by giving formal attention to patients' individual context and preferences. Furthermore, guidelines and medical education should pay more attention to patient perspective and how to deal with uncertainty, moving away from the current rationalistic approach. 


\section{Future research}

This study was executed in a single department with a specific group of doctors in a highly specialised field of care. More ethnographic studies should be performed to validate study findings in other settings. Future interventions for promoting SDM could then be tailored according to study findings. Implementation studies should further test whether the proposed changes in field and capital indeed lead to a better execution of SDM principles.

Acknowledgements The authors want to thank the doctors and nurses working at the Radboudumc gynaecological oncology department for participating in this study.

Contributors LS: designing the work, acquisition of data, analysis of data, interpretation of data; main drafting of the report; final approval of version to be published; accountable for all aspects of the report. JA: designing the work, interpretation of data; critically revising the report; final approval of version to be published; accountable for aspects of the report. SV: designing parts of the work, acquisition of data, analysis of data, some interpretation of data; minor revisions of the report; final approval of version to be published; accountable for aspects of the report. DB: partially involved in designing the work, interpretation of data; critically revising the report; final approval of version to be published; accountable for aspects of the report. TG: designing the work, supervising analysis of data, interpretation of data; critically revising the report; final approval of version to be published; accountable for aspects of the report. FS: designing the work, interpretation of data; critically revising the report; final approval of version to be published; accountable for aspects of the report.

Funding The authors have not declared a specific grant for this research from any funding agency in the public, commercial or not-for-profit sectors.

Disclaimer The views expressed in the submitted article are our own and not an official position of the institution.

Competing interests None declared.

Patient and public involvement Patients and/or the public were not involved in the design, or conduct, or reporting, or dissemination plans of this research.

Patient consent for publication Not required.

Ethics approval This study was judged by the regional medical ethical assessment committee and the local Radboudumc research ethics board (File number CM0:2017-3887).

Provenance and peer review Not commissioned; externally peer reviewed.

Data availability statement № data are available. Data consists of writtenout observations and other fieldnotes. Data are subject to interpretation and therefore not primarily open for publication.

Open access This is an open access article distributed in accordance with the Creative Commons Attribution Non Commercial (CC BY-NC 4.0) license, which permits others to distribute, remix, adapt, build upon this work non-commercially, and license their derivative works on different terms, provided the original work is properly cited, appropriate credit is given, any changes made indicated, and the use is non-commercial. See: http://creativecommons.org/licenses/by-nc/4.0/.

Author note Study results will be disseminated to all study participants (doctors, nurses).

\section{ORCID iD}

Laura Spinnewijn http://orcid.org/0000-0002-8708-2744

\section{REFERENCES}

1 Charles C, Gafni A, Whelan T. Shared decision-making in the medical encounter: what does it mean? (or it takes at least two to tango). Soc Sci Med 1997;44:681-92.

2 Stiggelbout AM, Van der Weijden T, De Wit MPT, et al. Shared decision making: really putting patients at the centre of healthcare. BMJ 2012;344:e256.

3 van der Weijden T, van Veenendaal H, Drenthen T, et al. Shared decision making in the Netherlands, is the time ripe for nationwide, structural implementation? Z Evid Fortbild Qual Gesundhwes 2011;105:283-8.
4 Légaré F, Moumjid-Ferdjaoui N, Drolet R, et al. Core competencies for shared decision making training programs: insights from an international, interdisciplinary Working group. J Contin Educ Health Prof 2013:33:267-73.

5 Légaré F, Witteman HO. Shared decision making: examining key elements and barriers to adoption into routine clinical practice. Health Aff 2013;32:276-84.

6 Stacey D, Légaré F, Lewis K, et al. Decision AIDS for people facing health treatment or screening decisions. Cochrane Database Syst Rev 2017;4:CD001431.

7 Hauser K, Koerfer A, Kuhr K, et al. Outcome-Relevant effects of shared decision making. Dtsch Arztebl Int 2015;112:665-71.

8 Elwyn G, Frosch DL, Kobrin S. Implementing shared decisionmaking: consider all the consequences. Implement Sci 2016;11:114.

9 Wyatt KD, Branda ME, Anderson RT, et al. Peering into the black box: a meta-analysis of how clinicians use decision AIDS during clinical encounters. Implement Sci 2014;9:26.

10 Henselmans I, Van Laarhoven HWM, Van der Vloodt J, et al. Shared decision making about palliative chemotherapy: A qualitative observation of talk about patients' preferences. Palliat Med 2017;31:625-33.

11 Welch S. Understand physician culture to facilitate change. begin by engaging their scientific and competitive qualities. Healthc Exec 2010;25:92-5.

12 Bourdieu P. Outline of a theory of practice. Cambridge: Cambridge University Press, 1977.

13 Pieterse AH, Baas-Thijssen MCM, Marijnen CAM, et al. Clinician and cancer patient views on patient participation in treatment decisionmaking: a quantitative and qualitative exploration. $\mathrm{Br} J$ Cancer 2008;99:875-82.

14 Kroneman M, Boerma W, van den Berg M, et al. Netherlands: health system review. Health Syst Transit 2016;18:p. 1-240.

15 3goedevragen.nl. Available: https://3goedevragen.nl

16 van der Geest S, Finkler K. Hospital ethnography: introduction. Soc Sci Med 2004;59:1995-2001.

17 Spradley JP. Participant observation. New York: Holt, Rinehart and Winston, 1980: 195.

18 Gerrits T. Patient-centered IVF: bioethics and care in a Dutch clinic. In: Berghahn books, 2016.

19 Emerson RM, Fretz RI, Shaw LL. Writing ethnographic fieldnotes. In: Chicago guides to writing, editing, and publishing. 2nd ed. Chicago: The University of Chicago Press, 2011: 289.

20 Makoul G, Clayman ML. An integrative model of shared decision making in medical encounters. Patient Educ Couns 2006;60:301-12.

21 Lindqvist S, Duncan A, Shepstone L, et al. Development of the 'Attitudes to Health Professionals Questionnaire' (AHPQ): a measure to assess interprofessional attitudes. J Interprof Care 2005;19:269-79.

22 www.oncoline.nl. Available: https://www.oncoline.nl

23 Hightower R. Doing ethnography in an urban hospital emergency department setting: understanding how culture was related to emergency physician habitus, 2010.

24 Nairn S, Pinnock D. Pierre Bourdieu: Expanding the scope of nursing research and practice. Nurs Philos 2017;18. doi:10.1111/nup.12167

25 Collyer FM, Willis KF, Franklin M, et al. Healthcare choice: Bourdieu's capital, habitus and field. Current Sociology 2015;63:685-99.

26 Reay * D. 'It's all becoming a habitus': beyond the habitual use of habitus in educational research. Br J Sociol Educ 2004;25:431-44.

27 Sackett DL, Rosenberg WM, Gray JA, et al. Evidence based medicine: what it is and what it isn't. BMJ 1996;312:71-2.

28 Simpkin AL, Schwartzstein RM. Tolerating Uncertainty - The Next Medical Revolution? N Engl J Med 2016;375:1713-5.

29 Jin CJ, Martimianakis MA, Kitto S, et al. Pressures to "measure up" in surgery: managing your image and managing your patient. Ann Surg 2012;256:989-93.

30 Gulbrandsen P, Clayman ML, Beach MC, et al. Shared decisionmaking as an existential journey: aiming for restored autonomous capacity. Patient Educ Couns 2016;99:1505-10.

31 Hamilton DW, Heaven B, Thomson RG, et al. Multidisciplinary team decision-making in cancer and the absent patient: a qualitative study. BMJ Open 2016;6:e012559.

32 Restivo L, Apostolidis T, Bouhnik A-D, et al. Patients' non-medical characteristics contribute to collective medical decision-making at multidisciplinary oncological team meetings. PLoS One 2016;11:e0154969.

33 Diamond-Brown L. The doctor-patient relationship as a toolkit for uncertain clinical decisions. Soc Sci Med 2016;159:108-15.

34 Kunneman M, Marijnen CAM, Baas-Thijssen MCM, et al. Considering patient values and treatment preferences enhances patient involvement in rectal cancer treatment decision making. Radiother Oncol 2015;117:338-42. 
35 Lo M-CM, Stacey CL. Beyond cultural competency: Bourdieu, patients and clinical encounters. Sociol Health IIIn 2008;30:741-55.

36 Green J, Thorogood N. Qualitative methods for health research. Sage, 2018.
37 Diamond-Brown L. "It can be challenging, it can be scary, it can be gratifying": Obstetricians' narratives of negotiating patient choice, clinical experience, and standards of care in decision-making. Soc Sci Med 2018;205:48-54. 